\title{
INCIDENCIA DEL TIPO DE LIDERAZGO EN EL CLIMA LABORAL CASO: ÁREA DE CAJAS DE LAS AGENCIAS DE QUITO DE UNA ENTIDAD FINANCIERA
}

\author{
INCIDENCE OF THE TYPE OF LEADERSHIP IN THE LABOR CLIMATE \\ CASE: AREA OF BOXES OF THE QUITO AGENCIES OF A FINANCIAL ENTITY
}

\author{
Jessie Villacrés Almeida \\ UTE \\ essie.villacres@ute.edu.ec
}

Héctor López Paredes

UTE

hector.lopez@ute.edu.ec

\section{Resumen}

El presente artículo tiene como objetivo analizar el tipo de liderazgo que genera mayor influencia en el clima laboral, tomando como caso de estudio el área de cajas de las agencias de Quito de una entidad financiera privada dedicada a la banca, que se caracterizan por tener alta rotación, bajo reconocimiento y mantener procesos de operación estandarizados. Para el análisis del tipo de liderazgo se consideró como modelo de referencia el empleado por HayGroup, y para la medición del clima laboral el modelo empleado por el Institudo Great Place to Word (GPTW), los cuales fueron fortalecidos con criterios hallados en la revisión teórica, obteniendo como resultado, en primer lugar la identificación de los estilos de liderazgo presentes en cada agencia estudiada, en segundo lugar se reconocieron los componentes del clima laboral presentes en cada agencia, y, en tercer lugar se analizaron los rasgos de cada tipo de liderazgo identificado que influyen en el clima laboral de cada agencia.

Palabras claves: Ambiente de trabajo, clima laboral, empleado, entidad financiera, liderazgo, satisfacción laboral, trabajadores,

\begin{abstract}
The objective of this article is to analyze the type of leadership that generates the greatest influence on the work climate, taking as a case study the cashier area of the Quito agencies of a private financial institution dedicated to banking, which are characterized by having high rotation, under recognition and maintain standardized operating processes. For the analysis of the type of leadership, the one used by HayGroup was considered as a reference model, and for the measurement of the work climate the model used by the Great Place to Word Institute (GPTW), which were strengthened with criteria found in the theoretical review, obtaining as a result, in the first place the identification of the styles of leadership present in each agency studied, secondly, the components of the working climate present in each agency were recognized, and, thirdly, the characteristics of each type of leadership were analyzed. Identified that influence the work climate of each agency.
\end{abstract}

Keywords: Environment work, work climate, employee, financial institution, leadership, satisfaction job, workers,

Clasificación JEL: J24, M12, M54 


\section{Introducción}

La investigación realizada fue motivada por uno de los principales problemas expuestos por el Subgerente de Operaciones de la entidad financiera motivo de estudio en relación al alto índice de rotación de los cajeros, en donde, Cumbicos (2016) afirma que muchas de estas desvinculaciones se dan debido a que el personal contratado, en su mayoría es gente joven que ha decidido empezar su carrera laboral en el área de cajas, pero cuando deciden estudiar, el horario es un limitante para continuar trabajando. Según Domínguez (2016), Oficial de Selección del Banco, la falta de apertura de horarios para poder estudiar genera malestar dentro del área de cajas, pues muchos colaboradores perciben que no tienen oportunidades de crecimiento si trabajan en la institución, adicional de la presión generada por las responsabilidades propias del cargo respecto al manejo de dinero y el trato con clientes.

Con lo expresado el estudio parte de la interrogante de investigación ¿Cómo incide el tipo de liderazgo en el clima laboral en el área de cajas de las agencias de Quito en una entidad financiera? Para lo cual se platearon como objetivos:

1. Identificar los tipos de liderazgo que se encuentran en el área de cajas de las agencias de Quito

2. Identificar los factores generales que están influyendo en el clima laboral en el área de cajas de las agencias de Quito.

3. Relacionar sobre qué factores del clima laboral están influyendo los tipos de liderazgo en el área de cajas de las agencias de Quito.

El levantamiento de información se realizó mediante la aplicación de una encuesta orientada a identificar los tipos de liderazgo existentes en el área de cajas de las agencias de la entidad financiera en estudio, y, de una encuesta que permitió el diagnóstico del clima laboral, para posterior a ello realizar el análisis de correlación y determinar su nivel de incidencia.

\section{Marco referencial}

La investigación ha considerado como principales referencias estudios que consideran la influencia del liderazgo en el cima laboral en empresas de distintos sectores, para buscar evidencia empírica en la entidad financiera objeto de estudio. Chávez (2013), en su tesis, "La Influencia del Liderazgo en el Clima Organizacional, Análisis de la PYME Ecuatoriana", en la cual se evidencia una incidencia del liderazgo del $70 \%$ en el clima laboral, y una influencia directa en el alcance de objetivos y metas de un determinado equipo de trabajo, afirmando que en las PYME el ámbito de acción del liderazgo es más cercano que una empresa grande porque los equipos de trabajo son más reducidos, la comunicación es más directa y por lo tanto con una estrecha vinculación entre la gestión y la cultura de la empresa

En la tesis de Peñarreta (2014), "La influencia de los estilos de liderazgo en los niveles de satisfacción laboral de los empleados del GAD Municipal de Loja", se relaciona al liderazgo con la satisfacción laboral de sus empleados y en consecuencia con el clima laboral. El estilo dominante en su investigación $(85,8 \%)$ fue el equilibrado, el mismo que se adapta a un ritmo cómodo en el que no existe esfuerzo por mejorar resultados. Finalmente su estudio demostró que el liderazgo no impacta en el ambiente de trabajo, considerando que el objeto de estudio tiene distintos criterios de evaluación de la satisfacción laboral y el líder.

Nieves (2013), en su tesis, "Análisis de la relación clima organizacional y satisfacción laboral en trabajadores del hospital del IESS de Esmeraldas", confirma que los siguientes factores influyen positivamente en el clima laboral: 
- Comunicación interna

- Reconocimiento

- Relaciones interpersonales

- Toma de decisiones

- Adaptación al cambio

- Entorno físico

Herrán (2014), en su tesis, "Los estilos de liderazgo y su influencia en el clima organizacional del personal de la escuela técnica de aviación civil", determina que los estilos de liderazgo existentes y predominantes en la ETAC son el democrático, seguidos por el autocrático, lo que hace notar que amerita una reorientación de estos hacia los estilos transformadores, en donde la construcción de relaciones personales es de confianza y sobre todo se motiva la creatividad y la conformación de equipos de trabajo. Los líderes de la ETAC son parte fundamental en la obtención de los objetivos institucionales, por ende se menciona que el éxito o fracaso de una organización depende en un $85 \%$ de los niveles gerenciales.

Los mencionados estudios concuerdan en que dependiendo el tipo de organización los tipos de liderazgo pueden influir en el clima laboral, sin embargo; en ciertos casos el liderazgo no es un factor predominante para la satisfacción laboral, por lo que denota la necesidad de realizar la investigación en una entidad financiera y validar si el liderazgo es un factor clave y determinante en el clima laboral.

\section{Marco teórico}

En la investigación se consideró varios estudios relacionados al liderazgo en las organizaciones, así como las principales teorías relacionadas al clima laboral con la finalidad de poder determinar su nivel de correlación, en donde, un punto de partida es lo expresado por (Maxwell, 1998) respecto a que el liderazgo es un factor que influye directamente en el clima laboral de las empresas, señalando que este determina el nivel de eficacia de una persona.

Otra visión es la de Silíceo, Ángulo, y Silíceo (2001) quienes afirman que de la influencia del liderazgo depende el logro de un fin, entendiendo que su figura en una organización no es estático sino que se trata de un sistema dinámico de interrelación y reciprocidad entre el líder y sus seguidores, aspecto que al interior de las empresas se refleja en el clima o entrono que permite dicha interacción.

Respecto a los estilos de liderazgo Abarca (2004) en su libro menciona que entre más estilos de liderazgo pueda tener una persona, es mejor, dado que ello permite su adaptabilidad a distintos entornos de trabajo, se deben considerar estilos como el autoritario, afiliativo, democrático y de coaching, en favor de obtener mejores resultados, mejor desempeño y mejor clima laboral.

Una de las principales firmas consultoras en el campo de los Recursos Humanos (HayGroup) propone el siguiente inventario compuesto de seis estilos de liderazgo que incrementan el rendimiento del equipo con mayor eficacia:

$\checkmark$ Coercitivo: Exige un cumplimiento inmediato de los objetivos por parte de su equipo de trabajo.

$\checkmark$ Orientativo: Su objetivo es establecer una visión a largo plazo y trabajar el liderazgo con su equipo.

$\checkmark$ Afiliativo: Implantar confidencia y fraternidad 
$\checkmark$ Participativo: Lograr el consenso del grupo y concebir nuevas ideas

$\checkmark$ Imitativo: Ejemplifica lo que desea que su equipo efectué y realiza tareas basándose en estándares.

$\checkmark$ Capacitador: Se enfoca en el desarrollo profesional de sus colaboradores

Cuando se habla de liderazgo es necesario hablar de la creación de equipos. "Un equipo de trabajo es sin duda un grupo, pero no todo grupo de trabajo forma un equipo. Para serlo ha de actuar de una forma concreta que lo convierta como tal" Porret (2012)

El líder debe desarrollar capacidades que lo ayuden a generar confianza en su equipo. Debe tener auto confianza y auto conocerse pues así logrará entender las necesidades del grupo. Debe ser el precursor de un ambiente sano, en el que las personas se desarrollen en base del aprendizaje y en donde el error sirva únicamente para reinventarse y mejorar.

Para Subirán, en Guillen (2010), indica que las vigentes teorías sobre liderazgo demuestran que lo principal para ser un buen líder son las competencias emocionales más no la innatas, y que las mismas se pueden desarrollar en la medida en la que uno lo desee, siendo la actitud clave para mejorar, aprender y desarrollar dichas habilidades; otro enfoque es el de Miller1(999) y Watkin (2000) quienes coinciden en que los líderes que puntúan alto en Inteligencia Emocional tienen ventaja a la hora de responder con flexibilidad a los cambios en sus entornos, a la hora de producir y comunicar visiones inspiradoras y de generar entusiasmo y esperanza por sus ideas, a lo que Haro y García (2015) asocia con un mejor desempeño en construir relaciones, confrontar problemas con los empleados, gestionar el cambio y generar un mejor clima laboral

Se puede decir que el clima laboral es el conjunto de características que suceden en un determinado ambiente, que pueden ayudar o perjudicar el entorno de trabajo y a su vez el cumplimiento de objetivos.

Para Cuadra y Veloso (2007), los cimientos de un buen clima laboral se relacionan, en términos generales, con el adecuado desempeño de la organización, y más específicamente con los siguientes indicadores, entre otros: igualdad entre el trabajo y la vida familiar y personal, beneficios sociales, satisfacción en el lugar de trabajo y conformidad con el estilo de liderazgo del inmediato superior.

El instituto de GPTW (2012) manifiesta que los factores que pueden incidir en el clima laboral son:

$\checkmark$ Credibilidad: Comunicación, competencia, integridad

$\checkmark$ Respeto: Apoyo, colaboración, cuidado

$\checkmark$ Orgullo: Trabajo individual, equipo, organización

$\checkmark$ Camaradería: relaciones cercanas, hospitalidad, sentido de unidad

$\checkmark$ Imparcialidad: equidad, ausencia de favoritismo, justicia

Chiavenato (2009) hace referencia a que el clima organizacional está estrechamente ligado al grado de motivación de las personas, por lo cual cuando éstas se encuentran muy motivadas, el clima organizacional mejora y se traduce en relaciones satisfactorias, que se caracterizan por actitudes de ánimo, interés, colaboración irrestricta, etc., manifestando además que las teorías de la motivación son fuentes primarias para lograr que los miembros de una organización se sientan a gusto en su trabajo, siendo necesario el contemplar 4 puntos críticos para intervenir dentro de la organización:

$\checkmark$ Las recompensas monetarias

$\checkmark$ Las recompensas extra monetarias como el reconocimiento, el prestigio y el status. 
$\checkmark \quad$ El enriquecimiento de las tareas

$\checkmark$ La flexibilidad en el horario de trabajo

\section{Metodología}

La metodología empleada para la investigación considera un modelo de investigación descriptivo y correlacional pues se evidenció el grado de relación que existe entre las dos variables motivo de estudio, siendo importante señalar que la investigación no fue experimental pues no se influyó sobre las variables de la misma.

Los métodos utilizados fueron:

$\checkmark$ Método inductivo-deductivo: se partió de una idea general que se analizó desde hechos particulares.

$\checkmark$ Método cuantitativo: se obtuvieron datos de la aplicación de los cuestionarios desarrollados para la medición de las variables de estudio.

$\checkmark$ Método analítico: se descompuso los tipos de liderazgo y los factores de clima laboral para entender cada uno de ellos y después relacionarlos, estadísticamente.

Las variables analizadas fueron:

- Variable dependiente: Clima Laboral

- Variable Independiente: Tipo de liderazgo

Para el desarrollo se consideró un muestreo aleatorio simple, que abarcó a 55 colaboradores de 11 agencias de Quito - Ecuador de una entidad financiera (Ver tabla 1)

\section{Tabla 1}

Distribución de la muestra de investigación

\begin{tabular}{ll}
\hline Cargo & \# de Colaboradores \\
\hline Jefe Operativo & 11
\end{tabular}

\section{Cajero Financiero $\quad 44$}

Fuente: Estudio previo a la obtención del título de Magister en Gestión del Talento Humano, Villacrés Jessie, 2016

Es decir, se valoró el clima laboral de 44 cajeros financieros y se evidenció el tipo de liderazgo de 11 Jefes operativos.

Se realizaron dos cuestionarios para medir el clima laboral y el liderazgo, los cuales se generaron de la revisión teórica y los modelos de referencia, siendo sometidas a la prueba de Alpa de Cronbach con un resultado de 0,81 en ambos casos, lo cual indica que las encuestas cuentan con un índice alto de confiabilidad para su adecuada aplicación a la población de la investigación.

\section{Resultados}


De la herramienta aplicada a los jefes operativos de la entidad financiera objeto de estudio, se

obtuvo

como

resultado

el tipo de liderazgo que se encontraba en cada agencia, posterior se realizó el análisis del clima laboral en cada agencia y finalmente se concluyó que factores afectan directamente al clima y si el liderazgo tenía una fuerte influencia sobre el mismo

Los resultados del tipo de liderazgo fueron los siguientes:

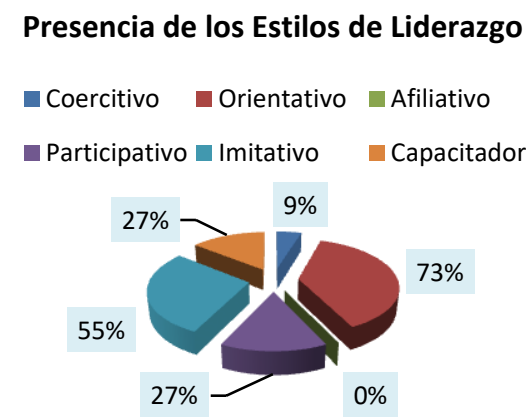

Figura 1 Presencia de los estilos de liderazgo en las agencias de la entidad financiera objeto de estudio, Quito - Ecuador 2016

En cuanto a clima se concluyó que existe orgullo por la organización en la mayoría de las agencias, siendo aspectos como remuneración y seguridad de empleo los que se encuentran puntuados bajo pues no son cubiertos de la manera en la que se desearía según los colaboradores.

En la siguiente tabla se puede evidenciar el tipo de liderazgo por agencia de acuerdo a la investigación realizada y que factores del clima son los mejor puntuados:

Tabla 2

Estilo de liderazgo por agencia estudiada y factor de clima mejor puntuado

\begin{tabular}{lll}
\hline Agencia & $\begin{array}{c}\text { Estilo de } \\
\text { liderazgo }\end{array}$ & $\begin{array}{c}\text { Factor de } \\
\text { clima } \\
\text { mejor } \\
\text { puntuado }\end{array}$ \\
\hline El Condado & Orientativo & Orgullo \\
\hline Alameda & Imitativo & $\begin{array}{l}\text { Ambiente } \\
\text { físico }\end{array}$ \\
\hline 12 de Octubre & Participativo & Supervisión \\
\hline Quicentro & Orientativo & Orgullo \\
Norte & & \\
\hline SMQ & Coercitivo & Orgullo \\
\hline El Inca & Orientativo & Orgullo \\
\hline Amazonas & Orientativo & Orgullo \\
\hline Quicentro Sur & Imitativo & Supervisión \\
\hline
\end{tabular}




\begin{tabular}{lll}
\hline El Recreo & Orientativo & Orgullo \\
\hline CCI & Orientativo & Orgullo \\
\hline San Luis & Orientativo & Orgullo \\
& & \\
\hline
\end{tabular}

(Villacrés, 2016)

A continuación se puede ver como se encuentra el clima laboral en cada agencia, considerando que 3 es muy satisfactorio, 2 satisfactorio, 1 poco satisfactorio y 0 insatisfactorio, y los estilos de liderazgo de cada agencia. Es así que evidenciamos que las agencias con el nivel más bajo en cuanto a satisfacción laboral poseen líderes Orientativos, al igual que las agencias mejor puntuadas, por lo que se comprueba que el estilo de liderazgo no influye necesariamente en el clima laboral del área de cajas, y que otros factores como remuneración, ambiente físico y orgullo pueden ser sobrevalorados al momento de una medición de clima laboral por parte de los colaboradores.

\begin{tabular}{|c|c|c|}
\hline Agencia & Clima Laboral & Estilo de Liderazgo \\
\hline Quicentro Sur & 2,66 & Orientativo \\
\hline El Inca & 2.50 & Imitativo \\
\hline Amazonas & 2.01 & Participativo-Imitativo \\
\hline El Condado & 2.50 & Orientativo-Imitativo-Capacitador \\
\hline Quicentro Norte & 2.47 & Coercitivo-Orientativo-capacitador \\
\hline Alameda & 1.88 & Orientativo-participativo \\
\hline 12 de Octubre & 2.22 & Orientativo \\
\hline SMQ & 2.12 & Imitativo \\
\hline El Recreo & 2.50 & $\begin{array}{l}\text { Orientativo-participativo-imitativo- } \\
\text { capacitador }\end{array}$ \\
\hline $\mathrm{CCl}$ & 1.93 & Orientativo-imitativo \\
\hline San Luis & 2.23 & Orientativo \\
\hline
\end{tabular}

Ilustración 1Estimación del clima laboral por agencia de la entidad financiera objeto de estudio y su estilo de liderazgo, Quito - Ecuador 2016

\section{Conclusiones}

Del estudio realizado se generan varios aportes a la ciencia, partiendo de señalar que la identificación del estilo de liderazgo en el área de cajas por agencia, no evidenció el estilo afiliativo, determinando que la falta de este componente genera la falta de identificación del personal con sus líderes.

Los estilos con mayor presencia en las agencias estudiadas son el Orientativo y el Imitativo, que muestran en el personal de cajas una mayor tendencia a cumplir disposiciones y limitar su iniciativa y proactividad en el trabajo.

El factor que influye positivamente en el clima laboral en el área de cajas de las agencias objeto de estudio es el orgullo, que se sustenta en la imagen generada por la institución financiera en el sector económico en el que se desempeña.

El factor que influye negativamente en el clima laboral del área de cajas de las agencias estudiadas es la remuneración, puesto que no se encuentra relación del personal entre sus responsabilidades y su reconocimiento económico. 
Los factores de liderazgo que están directamente relacionados sobre el clima laboral en cuanto al orgullo, el compromiso y el sentido de pertenencia, es el liderazgo de tipo orientativo seguido del imitativo, con lo cual la entidad financiera debe trabajar en la formación de líderes que sean conscientes de estos elementos para transmitir de manera positiva sus directrices respecto a lo que se debe hacer y cómo se lo debe hacer.

\section{Limitaciones}

A pesar que la investigación contó con una serie de pasos para evitar el sesgo en la información, debemos reconocer como limitaciones, en primer lugar el estudio fue transversal, siendo posible que varias respuestas sean consecuencia del efecto de una sola condición de tiempo; en segundo lugar los cuentionarios empleados fueron resultado de la propia investigación, y, a pesar modelos de referencia y la realización de pruebas estadísticas para su validación, consideramos que este debe ser replicado en otros estudios para fortalecer su utilidad.

Finalmente se reconoce que el no emplear de manera directa el nombre de la entidad financiera estudiada no permite diferenciar varios aspectos para el entendimiento de los resultados, sin embargo, cumplimos con mantener el nombre en reserva por pedido de la institución y rescatamos los resultados por su rigor en la construcción y validación de los instrumentos y en el planteamiento de los resultados.

\section{Bibliografía}

Abarca, N. (2004). Inteligencia emocional en el liderazgo. Santiago de Chile: Aguila Chilena de Ediciones S.A.

Chávez, M. (2013). La Influencia del Liderazgo en el Clima Organizacional, Análisis de la PYME Ecuatoriana. Quito.

Chiavenato, I. (2009). Motivación. En I. Chiavenato, Comportamiento Organizacional. La dinámica del éxito en las organizaciones (págs. 261-262-263). México DF: McGraw Hill.

Cuadra, A., \& Veloso, C. (2007). Liderazgo, Clima y Satisfacción Laboral en las Organizaciones. Universum, 40-56.

Cumbicos, M. (04 de Marzo de 2016). (J. Villacrés, Entrevistador)

Dominguez, M. B. (04 de Marzo de 2016). (J. Villacres, Entrevistador)

GPTW. (Junio de 2012). http://ftp.eeq.com.ec/upload/empresas-publicaseficientes/Cultura\%20Empresarial/Mauricio\%20Morillo.pdf. Obtenido de http://ftp.eeq.com.ec/upload/empresas-publicas-

eficientes/Cultura\%20Empresarial/Mauricio\%20Morillo.pdf

Guillen, C. (2010). El 90\% del éxito de un líder depende de su inteligencia emocional. Las competencias emocionales pueden desarrollarse. Gestión Práctica de Riesgos Laborales, 12-15.

Haro, J., \& García, M. (2015). Inteligencia Emocional Y Liderazgo Transformacional En Una Muestra De Directivos Españoles: Un Estudio Exploratorio. . Ansiedad y Estrés, 71-81.

HayGroup. (s.f.). http://atrium.haygroup.com/es/our-products/leadership-how-itworks.aspx.

Hernández, R., Fernández, C., \& Baptista, P. (1991). Metodología de la Invesigación. México: McGRAW - HILL .

Herrán, J. (2014). Los estilos de liderazgo y su influencia en el clima organizacional del personal de la escuela técnica de aviación civil. Quito. 
Nieves, J. (2013). Análisis de la relación clima organizacional y satisfacción laboral en trabajadores del Hospital del Iess de Esmeraldas. Quito.

Maxwell, J. (1998). Las 21 leyes irrefutables del liderazgo. Georgia: Maxwell Motivation, Inc.

Peñarreta, D. (2014). La influencia de los estilos de liderazgo en los niveles de satisfacción laboral de los empleados de GAD Municipal de Loja. Loja.

Porret, M. (2012). Gestión de personas. Manual para la gestión del capital humano en las organizaciones. Madrid: Esic.

Silíceo, A., Ángulo, B., \& Silíceo, F. (2001). Liderazgo: El Don de Servicio. México: McGraw Hill.

Villacrés, J. (22 de Marzo de 2016). Distribución de la población y muestra de investigación. Quito, Pichincha, Ecuador. 